\section{Bioanalysis in Latin America: where are we and where are we going?}

"Regulatory agencies in Latin America are trying to amend their guidelines to the international standard. In the near future, most of the countries will adopt specific requirements for generic medicines and this fact will allow the bioanalysis community in this region to expand.
Keywords: ANMAT $\approx$ ANVISA $\approx$ Bioanalytical Method Validation $\approx$ COFEPRIS $\approx$ guidelines " harmonization $=$ Latin America

Bioanalysis (BA) has experienced very fast growth in Latin America (LATAM). The first BA guidelines were implemented in the region in 1998, starting in Mexico and Brazil. More than 12 years later, Brazil is leading the BA market, both in number of assays and regarding the technology used. Market size as well as governmental policies for generic drugs can explain this. Most of the BA laboratories in Brazil currently use LC-MS/MS as the most common analytical technique. Mexico has decided to close its BA market to foreign bioanalytical CROs before such 'restraint of trade' occurs. Other countries such as Argentina, Chile and Uruguay are also trying to establish bioanalytical laboratories. In most LATAM countries, BA is mostly related to the analysis of small molecules in biological samples for bioequivalence studies. Other in vivo assays (e.g., drug metabolism and pharmacokinetics or preclinical tests) that require chemical analysis are very rare. The number of BA assays in Brazil is significantly higher compared with the rest of the countries in the region; the Brazilian National Health Surveillance Agency is the most important regulatory agency in the region. Although in LATAM most of the guidelines follow the US FDA's Bioanalytical Method Validation (BMV) guideline, documentation and quality assurance issues should still be addressed for global harmonization purposes.

Bioanalysis in LATAM has grown very fast in the last decade. Several countries have updated their surveillance regulations for generic medicines. In order to be registered as generic medicines, it is necessary to demonstrate in vitro and in vivo bioequivalence $(\mathrm{BE})$ towards the innovator. In vivo demonstration of $\mathrm{BE}$ requires chemical analysis of the pharmaceutical compounds in biological fluids. This fact has created a unique situation for the development of $\mathrm{BA}$ in this region.

For a new drug application, innovator companies should not follow the same rules for performing in vivo tests. Innovator companies in LATAM countries apply for registration using the data generated during the different phases (preclinical and clinical) of pharmaceutical development. BA of samples from preclinical and clinical phases are very rare in LATAM.

In accordance with this feature, one can expect that BA in LATAM would be more related to the analysis of biological samples for the analytical part of BE studies. Since the needs of generic pharmaceutical companies are more focused on obtaining the registration for their generic medicines, BA laboratories are engaged in analysis for this purpose.

It is worth mentioning that the Pan American Health Organization is supporting several projects for harmonization within the region by sharing the experiences among regulatory agencies and also training technicians for evaluating the applications (abbreviated new drug application).

\section{BA in LATAM}

Although each country in LATAM independently sets up its own regulatory framework, most of them followed the international regulation in the matter, based on the International Conference on Harmonization, FDA [1] and EMA [2] guidelines. Most regulatory agencies require the universal parameters of the BMV, such as specificity/selectivity, linearity, accuracy/precision, recovery, stability in biological matrix and the use of a validated method in the routine.

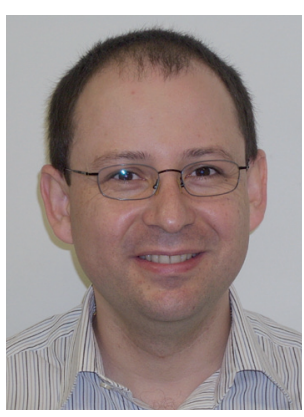

\section{Rafael Eliseo}

Barrientos-Astigarraga

Magabi Pesquisas Clínicas e Farmacêuticas Ltda. Av. Nações Unidas, 22.215, Jurubatuba, São Paulo, 04795-100, Brazil

Tel.: +55 II5 52। 0232

Fax: +55 II5 52। 0232

E-mail: rafael.barrientos@

magabi.com.br 
The differences rely on how to express results and the lack of requirements for some specific tests such as matrix factor, incurred sample reanalysis and ligand-binding assays (LBAs) [3].

Brazil, Mexico and Argentina emerge as the most important players, basically due to their market size. The actual situation of each country regarding BA is described below. Other countries, such as Chile, Uruguay, Peru and Colombia are still trying to set up their own regulatory framework.

\section{BA in Brazil}

Although Mexico published the first guideline on BMV in 1998, Brazil started the process 1 year later by creating the National Health Surveillance Agency (ANVISA) [101]. The agency started by regulating the abbreviated new drug application for generic medicines and released several guidelines, including those on BMV. Since the first guideline in 1999, it has been reviewed three times. The last version, termed RE 899, was released in May 2003.

RE 899 is a guideline that focuses on bioavailability/BE studies. Pharmacokinetic studies (Phase I), therapeutic drug monitoring and LBA are still incipient in Brazil. Instead of frequently revising its guidelines, ANVISA prefer to release documents called 'clarifications on requirements of RE 899', which clarify any specific points. A new draft, including new topics and other types of assays (pharmacokinetic and LBA), is expected to be released by the middle of 2011.

All the BA laboratories are inspected yearly by ANVISA for GLP purposes. Only certified laboratories and CROs, called Bioequivalence Centers, can conduct BA for legal purpose. It is important to highlight that all the 21 certified laboratories are equipped with at least one LC-MS/MS system, which means that this technique is well spread and consolidated among the BA community.

The National Health Surveillance Agency's technical staff (either reviewers or inspectors) have improved their skills for inspections and for the assessment of applications. ANVISA has also attended several BA meetings to share its experience. According to unpublished statistics, in 2009 more than $400 \mathrm{BE}$ studies were conducted in Brazil.

On the other hand, BA laboratories have been trying to set up discussion forums and, in 2007, the Brazilian Association of Bioequivalence Centers (ACBio) was founded [102]. At ACBio there is a technical group for discussion of bioanalysis. ACBio also organizes meetings and workshops for the BA community.

\section{BA in Mexico}

The first guideline (NOM-177-SSA1-1998) on BMV in LATAM was published in Mexico in 1998, and remains unchanged today. No revisions have been performed, and therefore, at present, it is considered as not harmonized with the rest of the countries.

The Federal Commission for the Protection against Sanitary Risk (COFEPRIS) is in charge of regulating BA laboratories [103]. According to a list published on its website, there are 20 BA laboratories certified in Mexico. COFEPRIS inspects them every 2 years. BA using LC-MS/MS as an analytical technique is still growing and it is expected to have only a few laboratories (four to five) using it. There are neither BA forums nor a representative organization in this country.

According to Mexican requirements, all steps for a BE study should be conducted inside the country. A 'restraint of trade' is likely to rise, which means that BA laboratories outside the country could be authorized to perform analysis by Mexican authorities.

\section{BA in Argentina}

The National Administration of Food, Medicines and Medicinal Technology (ANMAT) is the regulatory body in Argentina [104]. The guidelines for BMV (Provision 4844/2010) were recently revised in 2010. ANMAT does not have a certification program and it is not possible to find a list of BA laboratories. However, ANMAT do make random inspections into BA laboratories as needed.

Most BA laboratories are focused on academic issues. According to unpublished statistics, only three to four laboratories are routinely using LC-MS/MS as a bioanalytical tool.

There are neither BA forums nor a representative organization in this country.

\section{BA in Chile \& Uruguay}

The BA in these countries is almost incipient, but it exists.

Chile started publishing its guideline in 2005 (Provision 727/2005). The regulation of BA is related to the Public Health Institute (ISP), which is in charge of authorizing, controlling and certifying BA laboratories for performing bioanalysis [105]. There is one laboratory certified for legal purposes in Chile. 
The situation in Uruguay is very similar to Chile. The guideline for BMV follows the Decree No 12/2007 released by the Ministry of Public Health (MSP), which performs inspections and certifies BA laboratories [106].

The LC-MS/MS technique for performing $\mathrm{BA}$ is not yet used in these countries.

\section{BA in the rest of LATAM}

The remaining LATAM countries are still trying to set up a regulatory framework for BA. Countries such as Peru and Colombia are attempting to release their guideline on $\mathrm{BE}$ studies. It might be the start of BA in these countries.

\section{The Global Bioanalysis Consortium \& LATAM countries}

The Brazilian Association of Bioequivalence Centers has recently received an invitation for indicating a representative to take a seat on the Steering Committee of the Global Bioanalysis Consortium [4,107]. This step represents the first approach for inserting Brazilian and LATAM bioanalysts into a worldwide forum for harmonization.

The main purpose of this consortium is "to bring together stakeholders from the pharmaceutical industry, CROs and academia to share current understanding of bioanalysis guidelines, identify differences in these guidelines or differences in the interpretation or application thereof to routine regulated bioanalysis" [107]. Hopefully, LATAM bioanalysts can contribute to building bridges between regulatory agencies in each country in order to achieve this goal.

\section{Future perspective}

Regulatory agencies in LATAM are trying to amend their guidelines to meet the international standard. In the near future, most of the countries will adopt specific requirements for generic medicines and this fact will allow the BA community in this region to expand.

The National Health Surveillance Agency is becoming an important 'player' and might lead this process of expansion, influencing other regional agencies (e.g., COFEPRIS, ANMAT, ISP or MSP). Definitely, Brazil is a step ahead if compared with the rest of LATAM in terms of number of assays conducted, technology already implemented and market size.

There are some issues that should be more thoroughly discussed for sustained development, such as quality assurance processes, good laboratory practice and how to report data (documentation).

The Global Bioanalysis Consortium and other harmonization initiatives can help LATAM countries to better set up their guidelines and to move forward in a more rational way.

\section{Acknowledgments}

The author acknowledges the contacts in each country who provided all the information on the local perspective: Chile - Iván Saavedra and María Nella Gai; Mexico - Oscar Alderete, Gabriel Marcelín Jimenez and José Manuel Cárdenas; Argentina - Mario Dominguez and Miguel Vago; Uruguay - Mónica Cedrés; and Peru - Ofelia Villalva. The author also acknowledges Carlos Sverdlofffor revising this text.

\section{Financial \& competing interests disclosure}

The author has no relevant affiliations or financial involvement with any organization or entity with a financial interest in or financial conflict with the subject matter or materials discussed in the manuscript. This includes employment, consultancies, honoraria, stock ownership or options, expert testimony, grants or patents received or pending, or royalties. No writing assistance was utilized in the production of this manuscript.

\section{Bibliography}

1 US FDA. Guidance for Industry: Bioanalytical Method Validation. US Department of Health and Human Services FDA, Center for Drug Evaluation and Research (2001).

2 EMA. Guideline on validation of bioanalytical methods. EMA, London, UK (2009).

3 Savoie N, Garofolo F, van Amsterdam P et al. White Paper on recent issues in regulated bioanalysis and global harmonization of bioanalytical guidance. Bioanalysis 2(12), 1945-1960 (2010).
4 van Amsterdam P, Arnold M, Bansal S et al. Building the Global Bioanalysis Consortium: working towards a functional globally acceptable and harmonized guideline on bioanalytical method validation. Bioanalysis 2(11), 1801-1803 (2010).

\section{- Websites}

101 National Health Surveillance Agency. www.anvisa.gov.br

102 The Brazilian Association of Bioequivalence Centers. www.acbio.org.br
103 The Federal Commission for the Protection Against Sanitary Risk. www.cofepris.gob.mx

104 The National Administration of Food, Medicines and Medicinal Technology. www.anmat.gov.ar

105 Public Health Institute. www.ispch.cl

106 The Ministry of Public Health. www.msp.gub.uy

107 Global Bioanalysis Consortium. www.globalbioanalysisconsortium.org 